\title{
Die Alterssicherung der Beamten in Frankreich
}

\author{
Otto Kaufmann
}

I. Staat und Verwaltung

1. Politische und verfassungsrechtliche Rahmenbedingungen

2. Der Aufbau des öffentlichen Dienstes
a) Der öffentliche Dienst des Staates
b) Der öffentliche Dienst der Gebietskörperschaften
c) Der öffentliche Dienst des Krankenhauswesens

II. Die Grundstruktur des Beamtentums

1. Allgemeines

2. Das Beamtenstatut

3. Die Prinzipien des Beamtentums

4. Die Laufbahn
a) Eintritt in den Dienst
b) Die Vergütung
c) Die Stellung des Beamten
d) Beendigung der Laufbahn

III. Die Alterssicherung

1. Nebeneinander verschiedener Sicherungssysteme

a) Das allgemeine System

b) Die Sondersysteme

2. Die Basisalterssicherung im öffentlichen Dienst

a) Die Alterssicherung der Beamten

aa) Angleichung an das allgemeine Alterssicherungssystem

bb) Die Versicherten

cc) Die Voraussetzungen zum Bezug der Leistungen

dd) Die Höhe der Leistungen

b) Leistungen an Hinterbliebene

3. Freiwillige und obligatorische Zusatzversicherung für den öffentlichen Dienst

4. Die Finanzierung und Besteuerung

IV. Zusammenfassende Bemerkungen: Angleichung der Alterssicherungssysteme 
Frankreich kennt ein beamtenrechtliches System der öffentlichen Verwaltung und eine besondere Alterssicherung für die Beamten. Mit der großen Rentenreform von 2003 wurden auch für das Alterssicherungssystem des öffentlichen Dienstes Maßnahmen beschlossen, die nicht unerhebliche Änderungen zur Folge haben. ${ }^{1}$

\section{Staat und Verwaltung}

\section{Politische und verfassungsrechtliche Rahmenbedingungen}

Die Bedeutung des öffentlichen Dienstes hängt wesentlich von der Stellung und der Bedeutung ab, die dem Staat zukommen. Diese sind traditionell sehr ausgeprägt, woraus folgt, daß dem öffentlichen Dienst in Frankreich eine herausragende Rolle zukommt. Zudem wird die Organisation des öffentlichen Dienstes, wie auch seine Ausgestaltung, insbesondere auch durch die Staatsform beeinflußt. Aus diesem Grund bietet sich eine Skizzierung des Staatsaufbaus an. Seit der französischen Revolution und der Erklärung der Menschen- und Bürgerrechte von 1789 ist die öffentliche Verwaltung dem Recht und zwar besonderem Recht ${ }^{2}$ - untergeordnet. Der öffentliche Dienst - fonction publi$q u e$ - wird von den einen als traditionell überkommene Besonderheit gewürdigt, von anderen jedoch in seiner jetzigen Ausgestaltung in Frage gestellt. ${ }^{3}$

Frankreich ist eine parlamentarische Zweikammerndemokratie, deren Exekutive weitreichende Befugnisse eingeräumt werden. Die Abgeordneten der Nationalversammlung (Assemblée nationale) werden direkt vom Volk, die Mitglieder des Senats mittelbar durch die grands électeurs gewählt. Das sind die gewählten Abgeordneten der Nationalversammlung, Delegierte der gewählten Vertreter in den Regions- und Departementsräten (Conseils régionaux und généraux) sowie Vertreter der Gemeinderäte. Der Präsident der Republik wird für die Dauer von fünf Jahren direkt vom Volk gewählt. Der Premierminister, der laut Verfassung die Regierungsgeschäfte führt, wird auf Vorschlag des Präsidenten der Republik von den Abgeordneten der Nationalversammlung gewählt.

Die Verfassung überträgt dem Staatspräsidenten, der oberster Dienstherr der Staatsverwaltung ist, weitreichende Kompetenzen, die von Präsident Nicolas Sarkozy aber de facto noch erweitert wurden, mit der Konsequenz, daß von der Verfassung vorgegebene Kompetenzzuständigkeiten in der Realität dieser nicht mehr voll entsprechen; allerdings

1 Loi n 2003-775 du 21 août 2003.

2 TC (Tribunal des conflits) Blanco vom 8.2.1873, Long, Weil, Braibant, Delvolvé, Genevais, Les grands arrêts de la jurisprudence administrative, 16. Aufl. 2007, S. 1.

3 Dazu s. auch Silicani, Livre blanc sur l'avenir de la fonction publique, 2008 (S. 13 ff.). Argumente für und gegen die fonction publique in ihrer jetzigen Ausgestaltung faßt zusammen: Peiser, Droit de la fonction publique, 2006. 
wurden auch einige Verfassungsänderungen vorgenommen. Diese Kompetenzverschiebung auf politischer Ebene kann auch Auswirkungen auf den öffentlichen Dienst zeitigen, z.B. durch die Verringerung des Anteils der Beamten. Das soll vor allem dadurch geschehen, daß Beamte, die aus dem aktiven Dienst ausscheiden, nicht mehr ersetzt werden. Solche, auf eine Entscheidung des Staatspräsidenten zurückgehende Maßnahmen betrafen immerhin 33 \% der Fälle im Jahr 2008 und wohl 44 \% im Jahr 2009.

Frankreich weist immer noch eine stark zentralistisch geprägte Verwaltungsstruktur auf. Als Folge der zu Beginn der 1980er Jahre begonnenen Dezentralisierungsmaßnahmen wurde die Dezentralisierung als Verwaltungsform des Staates 2003 in die Verfassung aufgenommen. Frankreich ist seitdem eine dezentralisierte Republik. ${ }^{4}$ Unterhalb der zentralstaatlichen Ebene bestehen die Regionen, die jeweils mehrere Departements umfassen. Diesen Gebietskörperschaften stehen Vertreter der Regierung (Präfekten) vor. Aber auch die gewählten Repräsentanten der Einwohner der Region und der ihr zugehörenden Departements prägen die Politik auf diesen Ebenen im conseil régional ${ }^{5}$ und im conseil général. Regionalisierungsbestrebungen haben zum Ziel, insbesondere den Gebietskörperschaften eine größere Autonomie zu übertragen. Die untere Ebene sind die Gemeinden, die Zusammenschlüsse bilden können. Die Regionalisierung ist in ihrer weitgefächerten Vielfalt eines der schwierigen Themen der Innenpolitik, die alle Bereiche der Gesellschaft und des Staates berührt. ${ }^{6}$ Das zeitigt einerseits Auswirkungen auf die Gewichtung im öffentlichen Dienst, weil die Gebietskörperschaften ihr eigenes Beamtensystem haben und dieses mit der Übertragung von Kompetenzen des Zentralstaates auf die Gebietskörperschaften zahlenmäßig an Bedeutung zunimmt. Diese Kompetenzaufteilung, die den Regionalisierungsbestrebungen bzw. der Stärkung der Regionen voll entspricht, hat andererseits zur Folge, daß neben regionalen Rechtsnormen auch zentralstaatliche Rechtsnormen auf regionaler Ebene Anwendung finden, wodurch die Komplexität eher noch verstärkt wird.

\section{Der Aufbau des öffentlichen Dienstes}

Die Regeln der öffentlichen Verwaltung werden durch das Verwaltungsrecht vorgegeben, das auch auf den Beamtenstatus Anwendung findet. ${ }^{7}$

4 Art. 1 der Verfassung (Loi n 2003-231 du 17 mars 2003).

5 Insbesondere im sozialen Bereich haben die Gebietskörperschaften wichtige Kompetenzen.

6 Es geht darum, den Regionen auch die (finanziellen) Mittel zur Verfügung zu stellen, die es ihnen erlauben, alle ihnen übertragenen und noch zu übertragenden Aufgaben und Kompetenzen auch durchführen zu können. Die Abschaffung der Départements ist erneut in der Diskussion - allerdings mit geringer Erfolgsaussicht.

7 Allgemein zum öffentlichen Dienst: Plantey, La fonction publique, Traité général, Litec, 2. Aufl. 2001; Auby u. a., Droit de la fonction publique, Etat-collectivités locales - Hôpitaux, 5. Aufl. 2005; Chapus, Droit administratif général tome 2, 15. Aufl. 2001; de Laubadère, A. Gaudemet, Traité de droit administratif, t. 2 (La fonction publique), LGDJ, 12. Aufl. 2001. 
Es gibt drei verschiedene Systeme des öffentlichen Dienstes; auf diese finden sowohl für den gesamten öffentlichen Dienst gültige Rechtsnormen als auch jeweils spezifische Anwendung. ${ }^{8}$ Diese drei Systeme des öffentlichen Dienstes beschäftigen insgesamt 5,2 Mio. Personen. Somit sind die Verwaltungseinrichtungen und der Staat die größten Arbeitgeber des Landes. Berücksichtigt sind hierbei die Personen, die in einem öffentlichen Dienstverhältnis stehen und deren Ernennung und Tätigkeit den beamtenrechtlichen Vorschriften entsprechen.

Der Staatsdienst stellt 48,5 \%, der öffentliche Dienst der Gebietskörperschaften $32 \%$ und der der Krankenhäuser 19,5 \% der im öffentlichen Dienst Beschäftigten. Das entspricht $21,3 \%$ der Beschäftigten. ${ }^{9}$ Anzumerken ist, daß in manchen Bereichen des öffentlichen Dienstes, darunter hauptsächlich im Schulwesen, nichtverbeamtete Personen (agents non titulaires) in großer Zahl insbesondere als Aushilfen beschäftigt werden. Beamte können auch an eine andere Dienststelle innerhalb oder außerhalb ihres Systems abgeordnet werden. Das ist in erster Linie für die Beamten des Staatsdienstes von Bedeutung, mit einem Schwerpunkt im Schulwesen, gefolgt vom Finanzwesen. In einem jährlich erscheinenden Bericht wird die aktuelle Situation des gesamten öffentlichen Dienstes dargestellt. ${ }^{10}$

\section{a) Der öffentliche Dienst des Staates}

Die fonction publique de l'Etat (FPE) ${ }^{11}$ beschäftigt die Hälfte aller Beamten. Diese üben ihren Dienst in den zentralen Verwaltungseinheiten (hauptsächlich Ministerien), in den dezentralisierten Diensten (Präfekturen, Rektorate und Direktionen des gesamten Bildungs- bzw. Erziehungswesens) sowie in den nicht selbständigen öffentlichrechtlichen Anstalten (établissements publics à caractère administratif, EPA) aus.

Ein Kurzüberblick mit weiterführender Literatur: Schwarze, Droit administratif européen, 2. Aufl. 2009, S. 113.

8 In Neukaledonien und Französisch-Polynesien bestehen eigene öffentliche Dienste, die aber ähnlich wie der öffentliche Dienst im europäischen Frankreich organisiert sind.

9 Stand 31.12.2006; s. Rapport 2007-2008, S. 247 ff.

Die Anzahl der Staatsbeamten verringert sich tendenziell, die des öffentlichen Dienstes der Krankenhäuser bleibt konstant, während der öffentliche Dienst der Gebietskörperschaften signifikante Zuwächse verzeichnet - allerdings werden in diesem öffentlichen Dienst auch vermehrt nichtverbeamtete Bedienstete (non titulaires) eingestellt. Diese Kategorie von Angestellten verringert sich leicht in den beiden anderen öffentlichen Diensten.

10 Ministère du Budget, des comptes publics et de la fonction publique, Rapport annuel sur l'état de la fonction publique, La Documentation française.

11 Loi n $84-16$ du 11 janvier 1984. 


\section{b) Der öffentliche Dienst der Gebietskörperschaften}

Ungefähr ein Drittel der Beamten sind in der fonction publique territoriale (FPT) tätig. ${ }^{12}$ Ihre Dienstherren sind die territorialen Einheiten wie Regionen, Departements, Kommunen und Zusammenschlüsse von Kommunen sowie die öffentlich-rechtlichen Einrichtungen dieser Einheiten, wie z.B. die kommunalen Zentren für soziale Aktionen. Der Gesetzgeber hat wegen der Zunahme des öffentlichen Dienstes der Gebietskörperschaften Vorschriften erlassen, die den Beamten für den Fall des Wechsels aus dem Staatsdienst in den Dienst der Gebietskörperschaften gewisse Garantien zusichern. ${ }^{13}$

\section{c) Der öffentliche Dienst des Krankenhauswesens}

Ungefähr $20 \%$ der Beamten gehören der fonction publique hospitalière (FPH) an. ${ }^{14}$ Sie üben ihren Dienst in den öffentlichen medizinischen und sozialen Einrichtungen, insbesondere in Krankenhäusern, Altenheimen, Einrichtungen der Kindersozialhilfe und anderen sozialen Einrichtungen, die nicht dem privaten Sektor angehören, aus.

\section{Die Grundstruktur des Beamtentums}

Die verschiedenen öffentlichen Dienste kennen jeweils eigene Verwaltungsvorschriften mit nationaler Geltung. ${ }^{15}$ Doch finden gemeinsame grundlegende Regelungen für alle drei öffentlichen Dienste und ihre Beamten Anwendung. Sonderregelungen bestehen zudem für Richter und Soldaten. 16

12 Loi no 84-53 du 26 janvier 1984.

Der öffentliche Dienst der Stadt Paris ist der FPT zugeordnet, kennt jedoch Besonderheiten in seinem Verwaltungswesen und wird nach den Regeln der drei öffentlichen Dienste organisiert. Das erklärt sich aus dem Status von Paris, das zugleich Stadt und Departement ist.

13 Art. 104 Loi n $^{\circ}$ 2004-809 du 13 août 2004. Bounakhla $u$. a., Les flux de personnels dans les trois fonctions publiques, Vue d'ensemble $\mathrm{n}^{\circ} 2$, Rapport annuel sur l'état de la fonction publique Vol. 1, Faits et chiffres, 2006-2007.

14 Loi n 86-33 du 9 janvier 1986.

15 Die allgemeinen Rechte und Pflichten der Beamten sind in Teil 1 des Statuts niedergelegt, die Vorschriften des Staatsdienstes in Teil 2, die der territorialen Dienste in Teil 3 und schließlich die der Krankenhäuser und anderer sozialer Einrichtungen in Teil 4.

16 Eingehender zum Beamtenrecht und auch zu seinen Prinzipien, in deutscher Sprache - allerdings nicht zur Struktur des öffentlichen Dienstes, die zwischenzeitlich grundlegend verändert worden ist - s. Jevy, Frankreich. Landesbericht, und Maestre, Die Grands Corps im französischen öffentlichen Dienst, in: Kaiser/Mayer/Ule (Hrsg.), Recht und System des öffentlichen Dienstes, 1973, S. 43, 83. 


\section{Allgemeines}

Der öffentliche Dienst ist hinsichtlich der Bediensteten, die alle den Status von öffentlich Bediensteten (agents publics) haben, in zwei große Kategorien aufgeteilt. So unterscheidet man zwischen den ",non titularisés" und den „titularisés“, wobei letztere die eigentlichen Beamten, die ,fonctionnaires" sind und erstere es sein können, wenn sie Beamtenanwärter werden. Die Beamten stellen die Mehrzahl der öffentlich Bediensteten dar. Im Verwaltungsrecht finden sich die durch die Rechtsprechung im Laufe der Zeit entwickelten Begriffsbestimmungen zum Beamtenstatus.

Die Definition konnte (und kann noch) nach strafrechtlichen Kriterien vorgenommen werden, weil einschlägige Bestimmungen des Strafgesetzbuches den Beamten einerseits in der Ausübung seiner Funktion in besonderem Maße schützen, aber andererseits für bestimmte Verfehlungen oder Amtsanmaßungen besondere Sanktionen vorsehen. Ganz allgemein enthält das Strafgesetzbuch Vorschriften Personen betreffend, die „mit hoheitlicher Macht ausgestattet sind“ (dépositaires de l'autorité publique) oder die „,mit der Durchführung einer öffentlichen Aufgabe betraut sind“ (chargées d'une mission de service public). Diese letzte Definition findet sich ähnlich im Zivilgesetzbuch, nach dessen Vorschriften Beamter ist, wer eine, fonction publique“ ausübt.

Die Definition des Begriffs der Beamtenschaft setzt sich zwar von den privaten Arbeitsbeziehungen ab, aber es kann eine gewisse Erosion der rein unilateralen Entscheidung des Dienstherrn festgestellt werden. ${ }^{17}$ Schließlich wird auch das französische Recht des öffentlichen Dienstes von gemeinschaftsrechtlichen Regelungen beeinflußt. ${ }^{18}$ Mit der Übernahme dieser Regelungen fiel auch der Grundsatz, daß die französische Staatsangehörigkeit in jedem Fall Voraussetzung für den Beamtenstatus ist.

\section{Das Beamtenstatut}

Der Beamtenstatus ist gesetzlich geregelt (statut général). ${ }^{19}$ Die Begriffsbestimmung des Beamtentums ergibt sich aus dem öffentlich-rechtlichen Statut. ${ }^{20}$

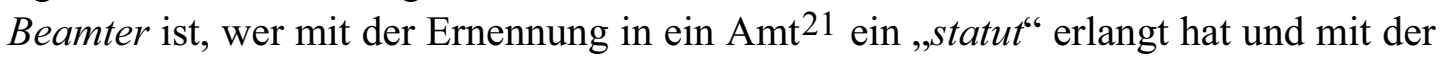
Verleihung einer bestimmten Amtsbezeichnung bzw. einem bestimmten Dienstgrad

17 Art. 96, Loi du 23 juillet 1987, Art. 5 Loi du 16 décembre 1996. Demhingegen stellt der Staatsrat (Conseil d'Etat, C.E.) fest, daß Beamte nicht einem Kollektivvertrag unterworfen sein könnten, C.E., Union des syndicats des affaires sociales, 11 décembre 1996, Rec. 983.

18 Dazu insbes. Sous la direction de G. Eckert, Y. Gautier, R. Kovar, D. Ritleng, Incidences du droit communautaire sur le droit public français, 2007; Lemoyne de Forges, L'adaptation de la fonction publique française au droit communautaire, 2003.

19 Loi du 13 juillet 1983.

20 Richter und das Militär unterliegen einem besonderen Status.

21 Wörtlich: ständig in einem dauerhaftem Beschäftigungsverhältnis - à titre permanent dans un emploi permanent. 
(grade) im öffentlichen Dienst aufgrund der Ernennung tätig ist (Eintritt in die Beamtenlaufbahn). Die Amtsbezeichnung (Dienstgrad), die dem Beamten persönlich verliehen wird, ist ihm eigen 22 und nicht an die effektive Dienstausübung bzw. Verwendung (emploi) gebunden.

Die Ernennung ist unabdingbare Voraussetzung, nur auf diese Weise kann der Beamtenstatus erlangt werden. Schließlich ist der Beamtenstatus von der hierarchischen Position unabhängig. Der statut garantiert die Sicherheit des Arbeitsplatzes, die Dienstausübung ist eine dauerhafte Verwaltungstätigkeit; das gilt für die gesamte Dauer des aktiven Dienstes. Die Ernennung gilt normalerweise für die Lebenszeit. Die Verwaltungsorganisation bestimmt die Dienstausübung, die Zahl der Dienstausübenden, die Art des Dienstes und die hierarchische Einteilung.

Jeder Beamte wird auf der Grundlage seines erlangten Diploms und seiner hierarchischen Einordnung für das Auswahlverfahren einer der Kategorien A, B oder C zugeordnet und ist mit Verwaltungs- oder Leitungsaufgaben betraut. ${ }^{23}$

Alle Beamten, die eine gleiche oder sehr ähnliche Funktion ausüben und eine ähnliche Karriere durchlaufen, gehören einem corps (Dienstgemeinschaft) an, das in vorgenannte Kategorien A, B oder C unterteilt ist. Eine Ausnahme davon bildet für bestimmte Dienste die Beamtenschaft des öffentlichen Dienstes der Krankenhäuser. Davon abgesehen, ist das corps die Grundlage der Organisation der Beamtenschaft und bleibt es für die Staatsbeamten. Sie vereinen alle Staatsbeamten, die ein bestimmtes Amt innehaben und die Voraussetzungen für die Ausübung bestimmter Dienste erfüllen. Vor allem die Beamten der Gebietskörperschaften sind seit den 1980er Jahren teilweise auch einem cadre d'emploi zugeordnet, ${ }^{24}$ der einerseits mehr Flexibilität zuläßt als ein corps, andererseits aber viel komplexer ist; die Zuordnung erfolgt nach der ausgeübten Funktion oder nach den Kriterien der Ernennung.

Die Verwaltung kann die Dienstausübung einseitig ändern, wogegen der Beamte kein Einspruchsrecht hat; im Gegenzug hat er innerhalb einer angemessenen Frist Anspruch auf Zuweisung in einen Dienst, der seinem Amt entspricht. Das verliehene Amt eröffnet seinem Inhaber das Recht, die Beamtenlaufbahn (carrière) zu durchlaufen. Der Aufstieg folgt bestimmten Regeln, insbesondere erfolgt durch die Hierarchie eine Bewertung.

In der Praxis ist häufig eine Unterscheidung zwischen dem Amt (Statut der Laufbahn), dem des Dienstes (ausgeübte Beschäftigung) und schließlich dem der Ausübung einer Funktion zu machen.

22 C.E. Dallier, 26 octobre 1945, Rec. 209: Der Beamte ist Eigentümer seiner Amtsbezeichnung.

23 Diese drei Kategorien sind im allgemeinen in Unterkategorien - ebenfalls aufgrund der Einstellung und des corps - unterteilt: A++ (hauts fonctionnaires, ernannt durch den Ministerrat), $\mathrm{A}+$ (höherer Dienst), A (Verwaltung), usw.

24 Art. 4 Loi nº 84-53 du 26 janvier 1984. 


\section{Die Prinzipien des Beamtentums}

Bestimmte Prinzipien finden auf den Beamtenstatus Anwendung, die neben den verwaltungsrechtlichen Vorschriften die Pflichten und Rechte der Beamten beeinflussen. Der Beamte befindet sich in einer „situation statutaire et réglementaire“, d.h. seine Rechte und Pflichten werden vom Gesetz vorgegeben, das grundlegende Garantien für die Beamten vorsieht. Artikel 34 der Verfassung bestimmt, daß allein der Gesetzgeber befugt ist, die grundrechtlichen Garantien der Zivil- und Militärbeamten (Berufssoldaten) des Staates festzulegen. ${ }^{25}$ Die Durchführungsvorschriften müssen auf Verordnungsebene ergehen. Der Dienstherr kann auch mit Zustimmung des Beamten die Arbeitsbedingungen individuell regeln, um sie zu verbessern oder unvorteilhafter zu gestalten, als dies von den anzuwendenden Rechtsnormen vorgesehen ist. Ein Beamter kann sich gegen Entscheidungen der Verwaltung, die seinen Status betreffen, auf die Verfassung berufen; dieses Privileg wird anderen Angestellten, die öffentlichem Recht unterliegen, nicht zugestanden. ${ }^{26}$

Der Beamte besitzt uneingeschränkte Gewissens- und Meinungsfreiheit. ${ }^{27}$ Er ist Bürger mit allen Rechten und hat das Recht, seine politische Meinung außerhalb des Dienstes kundzutun, auch wenn ihm eine gewisse Zurückhaltung auferlegt wird. Das wird mit der Neutralität des öffentlichen Dienstes begründet. Bestimmte Kategorien von Beamten, wie z.B. die Richter, müssen aufgrund ihrer besonderen Stellung und des besonderen rechtlichen Schutzes, der ihnen gegenüber der Hierarchie zugestanden wird, bei der Ausübung ihres Rechts auf freie Meinungsäußerung besondere Zurückhaltung üben und neutral bleiben. Das Neutralitätsgebot gilt auch für die Staatsräte als oberste Verwaltungsrichter. Die Ausübung gewerkschaftlicher Betätigung unterliegt ebenfalls der Beachtung dieser Verpflichtung. Es besteht Unvereinbarkeit zwischen der gleichzeitigen Ausübung des Dienstes und eines Mandats im französischen oder europäischen Parlament; in solch einem Fall gilt der Beamte als entsendet. Diese Unvereinbarkeit gilt nicht für Hochschullehrer und einige Berufskategorien in Alsace-Moselle. ${ }^{28}$

Im Gegensatz zum Recht auf gewerkschaftliche Betätigung ist das Streikrecht in keinem Gesetz niedergelegt, aber es ist ein Verfassungsrecht. ${ }^{29}$ Im Prinzip hat der Beamte das Recht zu streiken, um seine beruflichen Interessen zu verteidigen. Dabei ist das Streikrecht im Einklang mit der Pflicht zur gebotenen Zurückhaltung (devoir de réser-

25 Artikel 34 Verfassung: Durch Gesetz werden geregelt: (9) die den zivilen und militärischen Staatsbeamten gewährten grundlegenden Garantien.

26 C.E. Bellet, 18-6-1965. Rec. p. 370.

27 Dieses Prinzip ist im statut général niedergelegt (Art. 6 Loi du 13 juillet 1983) und ergibt sich zudem aus verschiedenen internationalen Abkommen.

28 Art. L.O. 142 Code électoral.

29 Conseil constitutionnel, C. const., Décision 79-105 DC, Rec. 33, RJCI-71, Favoreu/Philip, Les grandes décisions du conseil constitutionnel, 2001, S. 367. 
ve) auszuüben ${ }^{30}$, und die wesentlichen Aufgaben des öffentlichen Dienstes müssen weiter verrichtet werden können. ${ }^{31}$ Das Streikrecht wird im Rahmen der gesetzlichen Vorgaben ausgeübt; gegebenenfalls kann dies unter der Kontrolle des Richters auf dem Verordnungswege geschehen. ${ }^{32}$ Allerdings ist der Gesetzgeber befugt, bestimmten Kategorien von Beamten das Streikrecht zu untersagen; ein solches Verbot, das die Ausübung eines Freiheitsrechts betrifft, kann durch Gesetz geregelt werden. ${ }^{33}$ Entsprechende Gesetze wurden für die Richter, die Sicherheitskräfte (compagnies républicaines de sécurité), Polizei und Soldaten, das Gefängnispersonal, oder auch das mit Nachrichtenübermittlung beauftragte Personal des Innenministeriums verabschiedet. Ganz allgemein ist ein Streik insbesondere untersagt, wenn die öffentliche Ordnung (ordre public) in schwerwiegender Weise bedroht ist, die Sicherheit von Personen oder die Ausübung der Regierungsgeschäfte gefährdet sind.

\section{Die Laufbahn}

\section{a) Eintritt in den Dienst}

Im allgemeinen gründet sich eine Beamtenlaufbahn auf ein Auswahlverfahren (concours), das im Prinzip offen ist, aber auch intern organisiert sein kann. Außerdem sind Auswahlverfahren für Personen vorgesehen, die bereits Berufserfahrung außerhalb des öffentlichen Dienstes haben. Die Laufbahn beginnt mit der Einstellung als Praktikant. Mit Beendigung des Praktikums kann der Praktikant in das Beamtenverhältnis übernommen werden. Für den Aufstieg ist die zurückgelegt Dienstzeit von großer Bedeutung. $\mathrm{Zu}$ Beginn des effektiven Dienstes findet die Einsetzung des Beamten statt (installation), eine Formalität, die auch schriftlich durchgeführt werden kann und durch die der Beamte vor der Öffentlichkeit seine Tätigkeit tatsächlich aufnimmt. Beamte mancher corps bzw. solche, die mit bestimmten Aufgaben betraut sind, leisten vor Eintritt in den Dienst den Amtseid, der jedoch keinerlei politischen Charakter hat. ${ }^{34}$

\section{b) Die Vergütung}

Beamte haben das Recht, durch ihre gewerkschaftlichen Vertreter an Lohnverhandlungen teilzunehmen. Dies hat aber nur eine (sozial)politische Bedeutung, die Bezüge

30 C.E. 12 octobre 1956, Delle Coquand, Rec. 362; C.E. 5 juillet 1957, Cohen, Rec. 449.

31 C.E. 24 janvier 1961, Isnardon, Rec. 150.

32 C.E. 14 mars 1956, Hublin, Rec. 117.

33 C.E. Ass. 7 juillet 1950, Dehaene, Rec. 426; Long/Weil/Braibant/Delvolvé/Genevois, Les grands arrêts de la jurisprudence administrative, 16. Aufl. 2007, S. 420.

34 Das ist insbesondere der Fall für Richter, Offiziere und Unteroffiziere der Gendarmerie, für Beamte bei der Post, in der öffentlich-rechtlichen Buchhaltung und beim Zoll. 
werden vom Dienstherrn einseitig festgelegt. Die Höhe wird nach einem Punktesystem berechnet, die genaue Höhe für den Einzelnen ergibt sich aus der Gehaltsstufe. Zuzüglich zum Gehalt kann der Beamte eine Entschädigung erhalten, die sich aus der Zuordnung im corps oder dem cadre d'emploi sowie aus der ausgeübten Tätigkeit ergibt. Dieses als régime indemnitaire bezeichnete Zuschlagssystem wird auch „Prämie“ genannt. Dabei ist zwischen verschiedenen zusätzlichen Zahlungen zu unterscheiden. Persönliche Zuschläge können je nach der Familiensituation als Familienzuschläge geleistet werden. Vorgesehen sind auch Entschädigungszahlungen für Mehrarbeit. Dazu kommen zahlreiche diverse Zahlungen wie Ausgleichsentschädigungen, Überseeentschädigung (indemnité d'éloignement), Entschädigung für entstandene Kosten sowie Prämien und Sonderentschädigungen.

\section{c) Die Stellung des Beamten}

Ein Beamter ist im Normafall aktiv und übt eine seinem Amt entsprechende Tätigkeit aus. Mit seiner Zustimmung kann er in einen anderen Dienst abgeordnet werden; in diesem Fall wird er weiterhin von seiner ursprünglichen Dienststelle besoldet. Eine Versetzung ist auch gegen seinen Willen möglich, wenn es dienstlich geboten ist. Eine Versetzung aus familiären Gründen ist auf Antrag der oder des Betroffenen möglich. Der Beamte kann auch entsendet werden. In diesem Fall wird er von der Dienststelle besoldet, die ihn tatsächlich beschäftigt, aber die Laufbahnvoraussetzungen seines corps bzw. seiner ursprünglichen Dienststelle finden weiterhin Anwendung für die Karriere. Außerplanmäßiger Einsatz ist möglich. In diesem Fall finden insbesondere die Vorschriften über die Alterssicherung der effektiven Dienststelle Anwendung. Da ein Beamter zur besonderen Verwendung bzw. im einstweiligen Ruhestand keinen Dienst ausübt, sind die Vorschriften über die Alterssicherung und den Beamtenaufstieg nicht anwendbar. Schließlich können Vorschriften über den Wehrdienst und Erziehungsurlaub Anwendung finden.

\section{d) Beendigung der Laufbahn}

Die Laufbahn endet entweder

- mit Eintritt in den Ruhestand: Die Versetzung in den Ruhestand kann auf Antrag des Beamten geschehen, wenn er die notwendigen Voraussetzungen für eine Altersrente erfüllt, oder wenn er nicht mehr in der Lage ist, seinen Dienst auszuüben, oder aber durch Verrentung mit Erreichen des Renteneintrittsalters und schließlich durch eine disziplinarische Maßnahme des Dienstherrn,

- durch Quittierung des Dienstes,

- durch Kündigung wegen Dienstverweigerung oder durch Abberufung in anderen schwerwiegenden Fällen, oder

- mit dem Tod des Beamten. 


\section{Die Alterssicherung}

\section{Nebeneinander verschiedener Sicherungssysteme}

Die Alterssicherung ist nicht einheitlich organisiert, sondern wird von verschiedenen Alterssicherungssystemen getragen, die jeweils für bestimmte Personen- oder Berufsgruppen bzw. Tätigkeitsbereiche oder Branchen zuständig sind. Es bestehen sowohl Basissicherungen als auch obligatorische und freiwillige Zusatzalterssicherungen. Die Vielfalt der obligatorischen Alterssicherungssicherungssysteme hat zur Folge, daß ein Versicherter, der zwischen Berufssparten, die ein eigenes Sicherungssystem haben, wechselt, im Laufe seiner Versichertenzeit in mehr als einem Alterssicherungssystem versichert ist. Das kann auch für Beamte gelten. Manche Systeme wurden in früheren Reformschritten hinsichtlich des Leistungsbereichs und der Versicherungsvoraussetzungen an das allgemeine System angeglichen. ${ }^{35}$ Die große Rentenreform von 2003 zeitigt Auswirkungen auf das Sondersystem der Beamten, das immer mehr an das allgemeine Alterssicherungssystem angeglichen wird. ${ }^{36}$

\section{a) Das allgemeine System}

Das allgemeine soziale Sicherungssystem (régime général) hat als eigenen Sozialversicherungszweig ein Alterssicherungssystem (assurance vieillesse), das nach dem Umlageverfahren organisiert ist und durch eine obligatorische Alterszusatzversicherung ergänzt wird. ${ }^{37}$ In den persönlichen Geltungsbereich des régime général gehören die Beschäftigten von Industrie und Handel. ${ }^{38}$ Hier sei angemerkt, daß die Staatsbeamten und ihre Familien mindestens Anspruch auf die Sachleistungen haben, die im allgemeinen System der sozialen Sicherheit (régime général de sécurité sociale) vorgesehen sind. ${ }^{39}$ Das gilt für Leistungen bei Krankheit, Mutterschaft, Invalidität und Todesfall.

Ist die gesundheitliche Beeinträchtigung während der Ausübung des Dienstes eingetreten, so hat der Beamte Anspruch auf höhere Leistungen. Sonderregelungen sind für

35 Es sind dies die systèmes alignés. Die systèmes alignés sind die Alterssicherungssysteme der Landwirtschaft (MSA), die Systeme der Handwerksberufe und der Selbständigen sowie die Alterssicherung der freien Berufe. Vgl. Kaufmann, Die Reform der Alterssicherung in Frankreich: eine unendliche Geschichte, Die Angestelltenversicherung 2002, S. 329.

36 Kaufmann, Die aktuelle Reform der Alterssicherung in Frankreich 2004, Die Angestelltenversicherung 2004, S. 63; ders., Die Reform der Alterssicherung in Frankreich, DRdA 2/2004, S. 194.

37 Zum allgemeinen Alterssicherungssystem Kaufmann, Die französische Alterssicherung nach der Reform, ZFSH/SGB, 2004, S. 267; Lewerenz, Frankreich, in: Rentenversicherung im internationalen Vergleich, DRV-Schriften Band 45, 2003, S. 79.

38 Art. L. 712-1, D. 712-11 Code de la sécurité sociale, CSS.

39 Art. L. 712-1, D. 712-11 Code de la sécurité sociale, CSS. 
Beamte mit Wohnsitz außerhalb des französischen Mutterlandes vorgesehen. ${ }^{40}$ Allerdings werden die Leistungen für die Krankenkassen des allgemeinen Systems von der zuständigen lokalen mutuelle des fonctionnaires erbracht, was einer Verdoppelung des Verwaltungsaufwandes gleichkommt. ${ }^{41}$ Die Geldleistungen hingegen werden direkt von der zuständigen Verwaltung, d.h. vom Dienstherrn, erbracht. ${ }^{42}$ Der Dienstherr und der Beamte erbringen unterschiedlich hohe Beiträge (jeweils 9,7\% und 1\%). ${ }^{43}$

\section{b) Die Sondersysteme}

Sondersysteme (régimes spéciaux) gibt es für unselbständig Beschäftigte. Autonome Systeme (régimes autonomes), die zugleich Sondersysteme sind, sind zuständig für die Alterssicherung selbständig Beschäftigter. ${ }^{44}$ Es gibt zahlreiche weitere Sondersysteme für abhängig Beschäftigte in einer Vielzahl von Berufen. Diese sind vor allem für die Beschäftigten öffentlich-rechtlicher Unternehmen (entreprises à statut) zuständig. ${ }^{45}$ Auch das System des öffentlichen Dienstes ist ein Sondersystem.

Die im Jahr 2008 durchgeführte Reform der Sondersysteme hat zwar keine Angleichung der Sondersysteme an das Allgemeine bewirkt, aber eine Annäherung der Systeme, insbesondere was das Renteneintrittsalter angeht, das für Sondersysteme - wie übrigens auch für die Systeme des öffentlichen Dienstes - angehoben wurde. ${ }^{46}$

\section{Die Basisalterssicherung im öffentlichen Dienst}

Die Alterssicherungssysteme des öffentlichen Dienstes im engen Sinn - das régime spécial de retraite - erstreckt sich auf die Zivil- und Militärbeamten des Staates (fonctionnaires civiles et militaires), die Beschäftigten der Gebietskörperschaften (fonction publique territoriale) und auf die öffentlich-rechtlichen Krankenhäuser (fonction publi-

40 Art. L. 712-11 CSS.

41 Art. D. 712-6, Art. D. 712-30 CSS.

42 Art. D. 712-29 CSS.

43 Art. D 712-39, Art. D. 712-39 CSS.

$44 \mathrm{Zu}$ den Sondersystemen Kaufmann, Soziale Sicherheit in Frankreich, Teil 2: Sondersysteme und autonome Systeme, Soziale Sicherheit 1998, S. 299; ders., Alterssicherung in den französischen Sondersystemen, Die Angestelltenversicherung 1997, S. 430.

45 Das gilt insbesondere für die Elektrizitäts- und Gasunternehmen (industries électriques et gazières), für die Staatsbahn $(S N C F)$ sowie andere Eisen- und Straßenbahnen. Sondersysteme bestehen ebenfalls für das Personal des Senats, für die Mitglieder des Senats, Mitglieder der Nationalversammlung, das Personal der Nationalversammlung, für die Staatsbank (Banque de France), für die Beschäftigten der Pariser Oper, der Komödie (Comédie-Française), für die Beschäftigten des Straßburger Hafens, für den Klerus, für die Beschäftigten der Knappschaftskasse.

46 Tauran, Les réformes de 2008 des régimes spéciaux de sécurité sociale: un coup d'épée dans l'eau?, RDSS 2008, S. 748. 
que hospitalière). ${ }^{47}$ Es sind Sondersysteme, die Unterschiede zu den sozialversicherungsrechtlich ausgestalteten Schutzsystemen aufweisen.

Es bedarf jedoch für die Alterssicherung des Staatsdienstes keiner eigenen Organisation, weil der öffentliche Arbeitgeber, d.h. der Staat, die Bezüge - allerdings vermindert - im Alter weiter zahlt.

Für die Gebietskörperschaften und die öffentlich-rechtlichen Krankenhäuser ist das anders geregelt. Es besteht das régime spécial de retraites des agents des fonctions publiques territoriales et hospitalières, das von der Caisse nationale de retraites des agents des collectivités locales, CNRACL, verwaltet wird. ${ }^{48}$ Daneben gibt es schließlich das System der beim Staat beschäftigten Arbeiter sowie die Alterssicherung der nicht verbeamteten Angestellten der öffentlichen Dienste, die im Übrigen obligatorisch alterszusatzversichert sind.

\section{a) Die Alterssicherung der Beamten}

aa) Angleichung an das allgemeine Alterssicherungssystem

Die große Reformgesetzgebung von 2003 betraf in erster Linie das allgemeine Alterssicherungssystem, brachte aber auch für die Alterssicherung im öffentlichen Dienst grundlegende Veränderungen mit sich. Die im Reformgesetz vorgesehene Angleichung der Alterssicherung des öffentlichen Dienstes an das allgemeine System wird schrittweise vorgenommen und sollte bis 2012 zum großen Teil realisiert werden.

Da zwei völlig verschiedene Arten von Systemen gemeinsam reformiert und angeglichen werden sollen, war die Angleichung des Begriffs der „Versicherungsdauer“ im allgemeinen System und im System des öffentlichen Dienstes notwendige Voraussetzung, um eine allgemeine, systemübergreifende Versicherungsdauer von zunächst 40 Jahren herzustellen, um diese dann weiter zu verlängern. Es war unumgänglich, die Angleichung der Abschläge für vorzeitigen Rentenbezug im System des öffentlichen Dienstes und im allgemeinen System vorzunehmen.

\section{bb) Die Versicherten}

Der Ruhestand wird als Fortsetzung der aktiven Arbeitszeit verstanden. So spricht man folgerichtig auch von Ruhestandsgehalt (salaire d'inactivité) oder von der Weitergewährung der Bezüge (traitement différé). Die Altersrente wird, wie bereits dargelegt,

47 Hierzu Kessler/Moniolle, Le droit des retraites dans la fonction publique, 2000.

48 Ordonnance n 45-993 du 17 mai 1945. Die CNRACL erstellt einen jährlichen Rapport d'activité. Die Funktionsweise dieses Systems wird nicht gesondert dargestellt, weil es, abgesehen von der eigenen Kasse, weitgehend identisch ist mit dem der Staatsbeamten. Die auf diese Systeme anzuwendenden Vorschriften (Décret $n^{\circ}$ 85-1198 du 14 novembre 1985) stimmen mit denen des auf die Staatsbeamten anzuwendenden Code des pensions civiles et militaires de retraite überein. Insbesondere die Leistungsvoraussetzungen und die Leistungen sind identisch. Es wird somit diesbezüglich auf die Ausführungen zur Alterssicherung der Staatsbeamten verwiesen. 
aufgrund eines „Status“ gewährt. Versichert sind die Staatsbeamten, die im Staatsdienst aufgrund ihrer Ernennung dem Statut unterliegen und in einem Amt tätig sind. Die Leistungsbezieher müssen gemäß des Beamtengesetzes (statut général) aus dem aktiven Dienst auf ihr Verlangen hin oder gemäß der gesetzlichen Bestimmungen ausgeschieden sein. 49

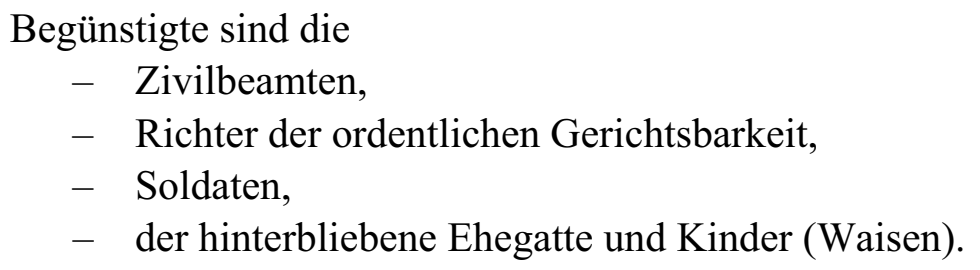

\section{cc) Die Voraussetzungen zum Bezug der Leistungen}

Die Versicherungsdaner für ein Ruhegehalt zum vollen Satz beträgt seit 2008, nach einer Übergangszeit nach der großen Reform von 2003, 160 Quartale, also 40 Jahre. Ziel ist die Dauer von 41 Jahren. Grundsätzlich werden für die Feststellung der Versicherungsdauer nur Zeiten aktiver Tätigkeit in einem Amt zugrunde gelegt.

Für die Erziehung während mindestens neun Jahren eines vor dem 1. Januar 2004 geborenen Kindes wird ein Versicherungsjahr zuerkannt, wenn der Dienst für mindestens zwei Monate unterbrochen wurde. Für Kinder, die nach dem 1. Januar 2004 geboren sind, werden bestimmte Unterbrechungen der Dienstzeit (Elternurlaub, Adoptionsurlaub, Dienstunterbrechung für die Erziehung eines Kindes unter acht Jahren, Teilunterbrechung der Dienstzeit) auf die Dienstzeit (Versicherungsdauer) angerechnet. Die Erziehung eines behinderten Kindes eröffnet ebenfalls Anspruch auf Anerkennung der Verlängerung der Dienstzeit. Beamte beiderlei Geschlechts können nach Erreichen der Mindestdienstzeit ein Ruhestandsgeld beziehen unter der Voraussetzung, die Dienstzeit tatsächlich unterbrochen zu haben.

Berücksichtigungszeiten gelten für Studienzeiten, 50 die im übrigen auch für die Höhe der Pension berücksichtigt werden, und für Perioden der Dienstunterbrechung wegen Kindererziehung. ${ }^{51}$

Das normale Ruhestandsalter ist auf 65 Jahre festgelegt, eine Alterspension zum vollen Satz kann jedoch ab dem 60. Lebensjahr bezogen werden; ${ }^{52}$ der Eintritt in den Ruhestand findet mit diesem, oft einem früheren Alter, statt. Beamte, die in einem der

49 Art. L. 3 Code des pensions civiles et militaires de retraite, C. pens. retr.

50 Art. L. 9 bis C. pens. retr.; Décret n ${ }^{\circ}$ 2003-1308 du 26 décembre 2003.

51 Art. L. 9, L. 12 bis C. pens. retr., s. auch Lanquetin, L'égalité entre hommes et femmes dans le régime spécial de retraite des Fonctionnaires, Droit social 2002, S. 178; Kaufmann, Frankreich: Altersgrenzen und Nachteilsausgleich in der Altersversicherung, ZIAS 2007, S. 135 (Schwerpunktheft Alterssicherung für Frauen in europäischen Ländern). Diesbezüglich scheinen trotz der aufgrund des Griesmar-Urteils des EuGH ergangenen Änderungen Beamtinnen noch rechtlich bevorteilt zu werden.

52 Art. 1er Loi n ${ }^{\circ} 84-834$ du 13 septembre 1984. 
„corps actifs" tätig waren, können sie ab dem 55. und einige Kategorien von Beamten $\mathrm{ab}$ dem 50. Lebensjahr beziehen. Diese beiden Altersgrenzen gelten für Beamte der „,catégorie B“, die in Ausübung ihres Dienstes besonderen Gefahren ausgesetzt waren oder deren Dienst besonders ermüdend war. ${ }^{53}$ Beamte, die mindestens 55 Jahre alt sind und 33 Beitragsjahre nachweisen können, haben die Möglichkeit des gleitenden Übergangs in den Ruhestand. 54

Keine Mindestaltersgenze für den Bezug eines Ruhegehalts gilt für Zivilbeamte, die drei Kinder oder ein behindertes Kind erzogen, aber die Mindestdienstzeit von 15 Jahren zurückgelegt haben.

Es ist eine Altersobergrenze vorgesehen, mit deren Erreichen der Beamte in den Ruhestand versetzt werden muß. Diese Altersgrenze ist auf 68 Jahre festgesetzt, ${ }^{55}$ ist aber nicht für alle Kategorien gleich und kann bis zum 70. Lebensjahr hinausgeschoben werden. Für bestimmte Kategorien gilt das 60. Lebensjahr als Ruhestandsalter.

Die Pensionsberechtigung besteht allerdings erst nach 15 effektiven Dienstjahren; bei Dienstunfähigkeit ist hingegen keine Wartezeit vorgesehen. ${ }^{56}$ Beamte, die keine 15 Dienstjahre nachweisen können, werden im allgemeinen System und im Zusatzsicherungssystem der Angestellten des öffentlichen Dienstes nachversichert.

\section{dd) Die Höhe der Leistungen}

Die Alterspension ist eine direkte finanzielle Zuwendung an Zivil- und Militärbeamte, die ihnen als Entlohnung für geleistete Dienste lebenslang gewährt wird. Die Höhe der Pension wird unter Berücksichtigung der hierarchischen Stellung, der Dauer und der Art des Dienstes festgesetzt und garantiert dem Berechtigten am Ende seiner Laufbahn die materiellen Voraussetzungen für ein Leben, das der Würde seines ausgeübten Dienstes entspricht. 57

Wenn die Voraussetzungen für eine Pension zum vollen Satz erfüllt sind, also 160 Quartale nachgewiesen werden können ${ }^{58}$, beträgt die Pension bis zu $75 \%$ der Höhe der Bezüge während der letzten sechs Dienstmonate. Berechnungsgrundlage für die Höhe der Pension sind somit die Bezüge der letzten sechs Monate vor Eintritt in den Ruhestand, wobei $2 \%$ davon angerechnet werden. ${ }^{59}$ Also: $2 \%$ (der letzten Bezüge) x Anzahl der Dienstjahre (mit der Obergrenze von $75 \%$ ).

53 Im Staatsdienst sind das Polizisten des aktiven Dienstes mit mindestens 25jähriger Dienstzeit und Justizvollzugsbeamte. Im Dienst der Gebietskörperschaften sind es unter Tage arbeitende Beamte der Abwasserwirtschaft.

54 Ordonnance $\mathrm{n}^{\circ}$ 82-297 du 31 mars 1982.

55 Art. 1er Loi n ${ }^{\circ} 75-1280$ du 30 septembre 1975.

56 Art. L. 4 C. pens. retr.

57 Art. L. 1 C. pens. retr.

58 Bis 2012 soll die Versicherungszeit auch im System des öffentlichen Dienstes auf 41 Jahre angehoben werden.

59 Wohingegen im allgemeinen System die vorteilhaftesten 25 Jahre zu Grunde gelegt werden. Die Regelung bis 2003: Die Staatsbeamten erhielten Anwartschaftsrechte von $2 \%$ der Bezüge für ein 
Unter bestimmten Voraussetzungen kann das Ruhegehalt allerdings durch den $\mathrm{Zu}-$ schlag von Bonifikationen bis zu 80 \% der letzten Bezüge ausmachen. Die Bonifikationen sind verschiedener Art und werden z.B. für Dienstausübung außerhalb Europas, oder aufgrund bestimmter familiärer Verhältnisse gewährt. ${ }^{60}$ Das Ruhegehalt wird für die Erziehung von drei Kindern über eine bestimmte Zeitdauer hinaus und vor dem 16. Lebensjahr der Kinder um $10 \%$ erhöht und um weitere $5 \%$ für jedes weitere Kind ab dem vierten.

Die Pension kann durch Verlängerung der Dienstzeit erhöht werden und wird vermindert, wenn bei Erreichen der Altersgrenze nicht die volle Versicherungszeit zurückgelegt worden ist. ${ }^{61}$ Dabei können bis zu 20 Quartale berücksichtigt werden. Wenn die Dienstzeit kürzer ist als die Anzahl von Quartalen, die für eine Pension zum vollen Satz notwendig ist, wird das Ruhegehalt seit 2006 um einen bis 2020 progressiv steigenden Prozentsatz pro fehlendem Quartal gekürzt (décote). ${ }^{62}$ Der Höchstsatz wird 1,25 \% betragen. Im umgekehrten Fall, wenn der Beamte mindestens 60 Jahre alt ist und eine größere Anzahl von Quartalen als die für ein Ruhegehalt zum vollen Satz notwendigen nachweisen kann, wird ihm eine Aufstockung des Ruhegehalts gewährt. Diese Aufstockung (surcote) wird bereits seit 2004 vorgenommen und beträgt 1,25 \% pro Quartal, was $5 \%$ pro Jahr entspricht.

Die Pension ist nicht pfändbar und darf nicht niedriger als das Lebensminimum sein, dessen Höhe durch Gesetz bestimmt ist. ${ }^{63} \mathrm{Im}$ Gegensatz zur aktiven Zeit werden im Ruhestand nur die Grundbezüge gezahlt. 64 Zuschläge und Sonderzahlungen werden nicht berücksichtigt. Aus diesem Grund ist das Ruhegehalt im Regelfall niedriger als die proportionale Minderung bezogen auf die Gesamtbezüge der aktiven Zeit.

Anders als im allgemeinen System ist kein Verbot des Bezugs eines Ruhegehalts neben einer Beschäftigung beim selben Dienstherrn vorgesehen; im Übrigen werden in einer Liste alle Tätigkeiten aufgezählt, für die kein Kumulierungsverbot gilt. Eine andere Liste sieht vor, daß bestimmte Arbeitgeber angeben müssen, für welche Ruhestandsbezieher sie Bezüge zahlen.

Die Leistungen werden nicht nur für die Alterssicherung, sondern auch bei Dienstunfähigkeit (Invalidität) erbracht. In diesem Fall muss keine Wartezeit nachgewiesen werden, und die Leistung wird altersunabhängig erbracht. Ist die Invalidität eine Konse-

Versicherungsjahr. Das führte nach 37,5 Jahren (d.h. nach der Versicherungszeit für eine Rente zum vollen Satz) zum Anspruch auf ein Ruhegehalt von 75 \% der Höhe der Bezüge der letzten sechs Monate. Nun müssen 40 Jahre nachgewiesen werden.

60 Siehe oben unter II. 4. b).

61 Art. L. 14 C. pens. retr.

622010 sind es noch 0,625 \%. Die Progression begann mit 0,125 \% ab 2006. Der Höchstsatz wird 2015 erreicht, wobei dann noch fünf Quartale wegfallen.

63 Art. L. 17 C. pens. retr.

64 Beamte zahlen die Sozialsteuer contribution sociale généralisée, CSG und die Sondersteuer zur Tilgung der Sozialschulden contribution au remboursement de la dette sociale, CRDS. 
quenz der Ausübung des Dienstes oder eines gemeinnützigen Einsatzes des Beamten wird diese Leistung neben dem Ruhestandsgehalt geleistet.

Beamte im Ruhestand, die ab dem 60. Lebensjahr eine Pension zum vollen Satz beziehen, können neben dem Ruhegehalt ein zusätzliches Arbeitseinkommen erzielen. Ab dem 65. Lebensjahr ist das auch neben dem Bezug eines gekürzten Ruhegehalts möglich.

Beamte mit Alterswohnsitz in Übersee erhalten einen Zuschlag zum normalen Ruhegehalt (indemnité temporaire de retraite des fonctionnaires de l'Etat outre-mer, ITR). ${ }^{65}$

Die Dynamisierung der Ruhegehälter im öffentlichen Dienst richtet sich wie im allgemeinen System nach der Preisentwicklung und nicht mehr nach den Bezügen der Aktiven; sie wird jährlich zum 1. April vorgenommen. 66

\section{b) Leistungen an Hinterbliebene}

Witwer und Witwen haben einen Anspruch auf eine Hinterbliebenenpension in Höhe von $50 \%$ des Ruhegehalts des Versicherten, wenn sie bestimmte, jeweils unterschiedliche Voraussetzungen hinsichtlich der Ehedauer erfüllen. ${ }^{67}$ Waisen erhalten unter bestimmten Altersvoraussetzungen eine Waisenrente. Stirbt der Beamte in Ausübung seines Dienstes, wird zusätzlich zur Hinterbliebenenpension ein Sterbegeld gezahlt.

Für einen Zeitraum von vier Jahren rückwirkend ab 2009 haben nun nicht nur Hinterbliebene, die mit dem Verstorbenen verheiratet waren, Anspruch auf Sterbegeld, sondern auch solche, die mit ihm mindestens zwei Jahre lang in einer eingetragenen eheähnlichen Gemeinschaft (PACS) gelebt haben. ${ }^{68}$

Beamte im Ruhestand (ausgenommen Richter, Arbeiter des Staates, Militäranghörige, Beamte der Post und France Télécom): 69

65 Morvan, La surpension des fonctionnaires retraités d'outre-mer: une fraude sociale sous les cocotiers, 2008, Point de vue, 230.

66 Art. L. 161-23-1 CSS. Vor der Reform war die Gehaltsstufenentwicklung im öffentlichen Dienst die Berechnungsgrundlage.

67 Die Dauer der Ehe muß vier Jahre betragen haben oder die Ehe muß zwei Jahre vor Eintritt in den Ruhestand geschlossen worden sein, es sei denn, sie ist nicht kinderlos geblieben.

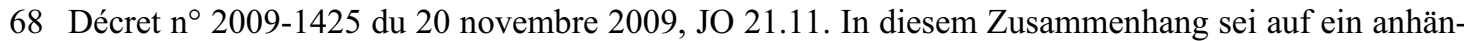
giges Verfahren vor einem Verwaltungsgericht hingewiesen, in dem die überlebende Lebenspartnerin die Zahlung einer Hinterbliebenenpension geltend macht. Sie verweist in der Sache ausdrücklich auf die Rechtssache EuGH vom 1. April 2008, C-267/06, Tadao Maruko / Versorgungsanstalt der deutschen Bühnen. Das Gericht hatte den Anspruch auf die Hinterbliebenenrente bejaht, insoweit die Leistung einem Entgelt entspricht. Analoge Anwendung auf das französische Recht würde zur Anerkennung der Leistung führen.

69 Stand 31.12.2007. S. Rapport annuel sur l'état de la fonction publique, Vol. 1, 2007-2008, S. 478. 


\begin{tabular}{|l|c|l|c|}
\hline & Staatsdienst & Gebietskörperschaften & Krankenhäuser \\
\hline Leistungen aus eigenem Recht & & & \\
\hline Gesamt & 1.110 .271 & 369.530 & 374.207 \\
\hline Davon Männer & 480.235 & 163.102 & 66.627 \\
\hline Hinterbliebenenleistungen & & & \\
\hline Gesamt & 277.545 & 95.468 & 40.957 \\
\hline Davon Männer & 33.007 & 9.135 & 11.113 \\
\hline
\end{tabular}

\section{Freiwillige und obligatorische Zusatzversicherung für den öffentlichen Dienst}

Da die Sozialversicherung für die konzeptionelle Auffassung der Alterssicherung der Beamten überflüssig ist, bestand in diesem System auch keine obligatorische Zusatzversicherung. Die große Rentenreform von 2003 sieht eine obligatorische Zusatzsicherung (Régime additionnel de la fonction publique, RAFP) vor. ${ }^{70}$

Durch diese Zusatzversicherung sollen die Nachteile, die manche Kategorien von Beschäftigten des öffentlichen Dienstes betreffen, weil bestimmte Gehaltsteile, die „primes“, für das Altersruhegehalt nicht berücksichtigt werden, zumindest teilweise ausgeglichen werden. ${ }^{71}$

Eine freiwillige Alterszusatzsicherung wird in Form eines Pensionsfonds (Préfon) angeboten. Dieser Fonds wurde 1964 von Gewerkschaften eingerichtet und wird von der nationalen Vorsorgekasse des öffentlichen Dienstes (Caisse Nationale de Prévoyance de la Fonction Publique, Préfon) verwaltet. Zugang haben aktive und ehemalige Beamte. 72

Schließlich gibt es das Complément retraite mutualiste de la fonction publique (COREM) und für den Krankenhausbereich das Comité de gestion des æeuvres sociales des établissements hospitaliers publice (CGOS).

\section{Die Finanzierung und Besteuerung}

Die Finanzierung der Alterssicherung wird durch das Staatsbudget gewährleistet. Dafür werden vom Staat 7,85 \% der monatlichen Bruttobezüge direkt einbehalten. Das

70 Art. 76 Reformgesetz, Décret n 2004-569 du 18 juin 2004.

71 Das Zusatzsicherungssystem des öffentlichen Dienstes erhielt eine Auszeichnung für den besten Finanzanleger (le grand prix de l'investisseur responsable), die 2008 von Natixis Asset Management und dem consultant Amadeis in Zusammenarbeit mit Les Echos geschaffen wurde. Mit dieser Auszeichnung soll der Finanzanleger bedacht werden, der bei seiner Tätigkeit am besten die Umweltbedingungen, die sozialen Gegebenheiten und eine gute Anlegestrategie miteinander in Einklang bringt.

72 Préfon zählt 400.000 Mitglieder, wovon 80.000 im Ruhestand sind. Die Reserven betragen 9 Mrd. Euro, die Einnahmen beliefen sich im Jahr 2008 auf 462 Mio. Euro. 
gilt als Beitragszahlung des Beamten. Diese Beiträge stellen eine Einnahme dar, die aber nicht zweckgebunden ist und nicht für die Auszahlung der Ruhegehälter benutzt wird, sondern ins Staatsbudget fließt. Der Beamte selbst entrichtet keine weiteren Beiträge. Die obligatorische Zusatzsicherung ist nach dem Umlageverfahren in einem Punktesystem organisiert. Die Finanzierung erfolgt aus Beiträgen, die auf Bestandteile der Bezüge der Beamten erhoben werden, die nicht für die Berechnung der Alterspension berücksichtigt werden. ${ }^{73}$ Die Beiträge von insgesamt $10 \%$ werden je zur Hälfte vom Dienstherrn und vom Beamten getragen. Die Berechnungsgröße beträgt $20 \%$ des Bruttoentgelts. 74

Das Ruhegehalt unterliegt der Einkommensteuer sowie den Sozialsteuern contribution sociale généralisée (CSG) und contribution au remboursement de la dette sociale (CRDS).

\section{Zusammenfassende Bemerkungen: Angleichung der Alterssicherungs- systeme}

Es wird bis 2020 eine weitgehende Harmonisierung zwischen dem allgemeinen System und den systèmes alignés einerseits und dem des öffentlichen Dienstes andererseits hergestellt. Einige Besonderheiten des Systems der Beamtenalterssicherung wurden bereits abgeschafft, andere werden in Zukunft verdrängt. Auf diese Weise wird das System der Beamten immer mehr dem allgemeinen Sicherungssystem angeglichen. Einer solchen Annäherung bzw. Harmonisierung steht nichts entgegen. Dennoch werden Besonderheiten des Sondersystems des öffentlichen Dienstes - eigentlich der drei öffentlichen Dienste - beibehalten, und es wird weiterhin besonderen Regeln folgen. Insbesondere deutet nichts darauf hin, daß das Beitragssystem des régime général für das Sondersystem übernommen werden oder die Leistungshöhe explizit vermindert werden soll. Eine ganz andere Frage ist die der Ersetzung mancher Beamtenstellen durch Arbeitnehmer.

Im allgemeinen System wird auf die Versicherungsdauer bzw. Beitragszeit abgestellt, während diese Elemente im System des öffentlichen Dienstes eine andere Bedeutung haben, weil dieses System, abgesehen von der Mindestdienstdauer von 15 Jahren, keine Versicherungszeit kennt. Im öffentlichen Dienst wurde die Versicherungszeit von 40 Jahren (2003 waren es 37,5) für eine Altersrente zum vollen Satz im Jahr 2008 durch eine schrittweise Erhöhung erreicht. Die bestehenden Unterschiede hinsichtlich des Renteneintrittsalters, des Mindestalters oder für die Weiterzahlung der vollen Bezüge erklären sich aus der Bindung des Ruhestandsgehalts an die konkrete berufliche Stel-

73 Das sind z.B. die Familienzulage, die Vergütung von Über- und Zusatzstunden, der geldwerte Vorteil materieller Zuwendungen und Leistungen.

74 Art. 2 Décret n² 2004-569 du 18 juin 2004. 
lung des Berechtigten zum Zeitpunkt des Eintritts in den Ruhestand. Für die Berechnung der Pensionshöhe wird auf die Dauer des Dienstes abgestellt sowie auf verschiedene Bonifikationen Bezug genommen, die sich dann in den ruhegehaltsfähigen Dienstjahren ausdrücken.

Für das System des öffentlichen Dienstes gilt nun ebenfalls das Element der Versicherungsdauer, das langfristig systemübergreifend sein wird. ${ }^{75}$ Das zeigt sich durch die Einführung eines progressiven Minderungsfaktors (décote) im Jahre 2006 an Stelle der vorherigen proportionalen Kürzung sowie der Möglichkeit, das Ruhegehalt zu erhöhen (surcote). Die Beamtensicherung lernt so ein flexibles System der Laufbahn kennen.76 Dieses Element bringt in den öffentlichen Dienst eine bislang nicht bekannte Flexibilisierung bezüglich des Eintritts in den Ruhestand. Allerdings wird die gewünschte Flexibilisierung nur Wirkung zeigen, wenn die Beamten wirklich ermutigt werden, länger im aktiven Dienst zu bleiben und es wirklich wollen.

75 Art. 51 Reformgesetz; Art. L. 14 Code des pensions civiles et militaires des retraites.

76 Dazu auch: Fonctions publiques de la réforme des retraites à la gestion des parcours professionnels, Rapport au Conseil d'orientation des retraites, Rapporteur Mme Marianne Berthod-Wurmser, 2006. 


\title{
Die Alterssicherung von Beamten in Italien
}

\author{
Eva Maria Hohnerlein
}

I. Einführung

II. Rechtliche Stellung der Beamten

1. Verfassungsrechtlicher Rahmen

2. Grundlagen des Dienstrechts

III. Die gesetzliche Regelung der Alterssicherung von staatlichen Beamten

1. Grundzüge der Alterssicherung im Rahmen der allgemeinen

Rentenversicherung für Arbeitnehmer
a) Voraussetzungen der Altersrente nach dem lohnbezogenen oder dem gemischten System
b) Voraussetzungen der Altersrente im (ausschließlich) beitragsbezogenen System
c) Voraussetzungen für eine „Beitragsaltersrente“ (pensione di anzianità)
d) Die Berechnungsregeln

2. Reformen zur Angleichung des Sondersystems für Beamte an die Regelungen des privaten Sektors
a) Materiellrechtliche Reformen der Alterssicherung im öffentlichen Dienst
b) Organisationsrechtliche Reformen

3. Die Regelungen zur Alterssicherung der Beamten
a) Leistungen
b) Allgemeine Altersgrenzen und Frauenaltersgrenzen
c) Allgemeine Leistungsvoraussetzungen
d) Berechnung und Leistungshöhe der Altersrente
e) Dienstaltersrente
f) Aufschub des Rentenbeginns bis 70

4. Finanzierung

5. Verwaltung

6. Besonderer Rechtsweg

7. Die Zusatzversorgung des öffentlichen Dienstes

IV. Bewertung

Statistischer Anhang 